\title{
Nuclear effects in neutrino induced reactions
}

\author{
M. J. Vicente Vacas, L. Alvarez-Ruso, L. S. Geng \\ Departamento de Física Teórica and IFIC, \\ Universidad de Valencia-CSIC, \\ 46100 Burjassot (Valencia), Spain \\ J. Nieves, M. Valverde \\ Departmento de Física Atómica Molecular y Nuclear, \\ Universidad de Granada, \\ 18071 Granada, Spain \\ S. Hirenzaki \\ Department of Physics, Nara Women's University, Nara, 630-8506, Japan
}

\begin{abstract}
We discuss the relevance of nuclear medium effects in the analysis of some low and medium energy neutrino reactions of current interest. In particular, we study the Quasi-Elastic (QE) process, where RPA correlations and Final State Interactions (FSI) are shown to play a crucial role. We have also investigated the $\nu$ induced coherent pion production. We find a strong reduction of the cross section due to the distortion of the pion wave function and the modification of the production mechanisms in the nucleus. The sensitivity of the results to the axial $N \Delta$ coupling $C_{5}^{A}(0)$ has been also investigated.
\end{abstract}

PACS numbers: 25.30.Pt, 13.15.+g, 23.40.Bw

Keywords: Neutrino-nucleus interactions; N- $\Delta$ form factors; Hadrons in nuclear medium.

\section{INTRODUCTION}

The analysis of the new generation of neutrino oscillation experiments requires a good knowledge of the neutrino nucleus cross sections. This was soon acknowledged and a series of workshops (Nuint) fully dedicated to this topic started in 2001. Even more interesting for hadron physics is the fact that the very high luminosity of the neutrino beams is starting to provide valuable information about the axial properties of baryons, that would be hard to obtain with electromagnetic probes.

These reasons have led to much theoretical work [1] studying the basic processes, but still many of the codes used in the experimental analysis apply outdated or incomplete models, or they are extrapolated out of their validity limits. For example, in Ref. 2] an artificial free parameter that modifies Pauli-blocking was introduced to fit the low $q^{2}$ QE data. In the study of coherent $\pi$ production of Ref. [3], some nuclear parameters were modified to unrealistic values. For instance, the Fermi momentum was varied up to values corresponding to almost four times the normal nuclear density. These kind of approaches may fit the data and not affect the most important observables, but still the introduction of wrong and uncontrolled physical assumptions in the models leads to some doubts about the reliability of the results.

In this talk, I will present a few selected results of the ongoing work of our group on QE scattering and coherent pion production induced by neutrinos.

\section{QUASIELASTIC NEUTRINO NUCLEUS SCATTERING}

This topic has been widely studied. See e.g. [4, [5, 6] and references therein. It is well known that the impulse approximation (IA) in a Fermi Gas (FG) model, frequently used in the analysis of neutrino experiments, fails to describe lepton nucleus cross sections. Other nuclear effects like the use of proper nucleon spectral functions and correlations are clearly needed [7]. Furthermore, the models should be tested on other processes which measure the nuclear response at similar excitation energies.

Here, we will discuss some of the results of Refs. [4, 8] on the QE $\nu$-nucleus process. This model had been successfully used in the study of photonuclear processes like $(\gamma, N)$ or $(\gamma, N \pi)$ 9, 10] and electronuclear reactions [1]. In these works, apart from the above mentioned nuclear effects implemented in a consistent many body framework, FSI is described by a MonteCarlo simulation. Many of the calculations use either the plane wave (PW) or the distorted wave impulse approximation (DWIA). Neither of them is appropriate for inclusive processes like QE. In PW, the strong interaction between the final nucleon and the residual nucleus is fully neglected. In the DWIA only the elastic part 
of this interaction is properly taken into account, whereas the inelastic processes are accounted for by means of the imaginary part of some optical potential that reduces the nucleon flux and thus, the cross section. However, in these inelastic processes, the nucleon simply changes energy, charge and/or angle and possibly more nucleons are emitted, still contributing to the QE cross section.

The main effect found for the inclusive $(\nu, l)$ reaction, at the intermediate energies where this model is better suited, is the large reduction of the cross section due to the RPA correlations as compared to the IA descriptions. Additionally, the use of realistic nucleon spectral functions distorts the shape of the QE peak producing a significant broadening. Concerning the processes where a nucleon is detected, the inclusion of the FSI means that, even for light nuclei, the nucleon spectrum is strongly displaced towards low energies. For CC events a significant cross section for the "wrong" charge nucleon appears. See Ref. [8] for a detailed discussion.

\section{COHERENT NEUTRINO INDUCED $\pi$ PRODUCTION}

A model for $\nu$ induced coherent $\pi$ production has been developed in Refs. [12, 13, 14]. This model improves upon previous calculations [15, 16] by using a more complete description of the elementary pion production and a better treatment of the pion FSI. The process is dominated by the $\Delta$ excitation, but other background pieces like the cross $\Delta$ and nucleon-pole terms are also considered. There are many other contributions, relevant for the reaction in a single nucleon [17], but, due to their isovector nature, they cancel for isospin symmetric nuclei and we neglect them. The purely nucleonic part of the hadronic current is better known and is written in terms of vector and axial form factors (FF). The vector FF are extracted from electron scattering data and the axial ones are contrained by PCAC. The $N-\Delta$ transition is more complex and PCAC is insufficient to fully determine the axial sector. The usually called Adler model, which takes $C_{3}^{A}=0$ and $C_{4}^{A}=-C_{5}^{A} / 4$ was chosen and two different parametrizations for $C_{5}^{A}$ [17, 18] which reproduce the available pion production data on the studied energy region [18, 19] were considered.

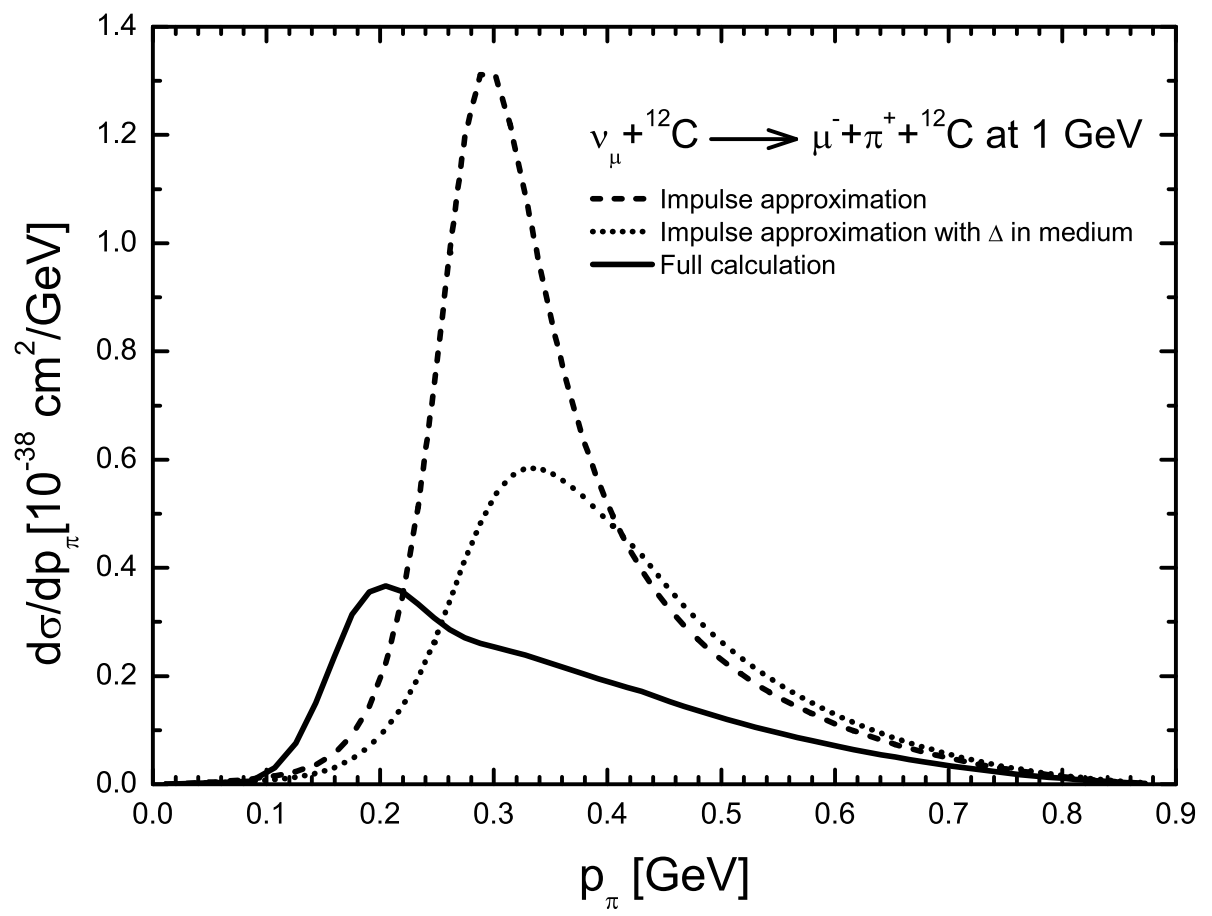

FIG. 1: Coherent pion production cross section.

The nuclear medium modification of the $\Delta$ properties is taken into account by modifying its propagator with the inclusion of an energy and density dependent selfenergy well tested in many $\pi$ nucleus processes. This effect, sometimes omitted in other calculations [20], reduces the cross section by around $40 \%$ for $1 \mathrm{GeV}$ neutrinos, (see 
Fig. 1). There are other nuclear effects like Fermi motion or Pauli blocking but the most important one is the FSI of the pion. It is implemented in the DW approximation by solving the Klein-Gordon equation with the optical potential of Refs. 21, 22]. We find that pion distortion moves the pion spectrum to lower energies and also reduces the cross section. One can notice a large reduction at the $\Delta$ resonance peak due to the large pion absorption at that energy.

The results are more sensitive to $C_{5}^{A}$ than in the case of the incoherent reaction. This can be understood from two facts. First, in the forward direction $\left(q^{2}=0\right)$, dominant in the coherent process, the only FF contributing to the $\Delta$ excitation is $C_{5}^{A}$ [23]. Second, many of the background terms cancel in the coherent sum of amplitudes. Thus, the coherent process is very sensitive to the relatively unknown $N \Delta$ transition axial FF [24, 25, 26, 27, 28] and could eventually provide valuable information complementary to what could be obtained from parity violating electroexcitation of the $\Delta[29]$.

\section{Acknowledgments}

This work was partially supported by MEC contracts FIS2005-00810, FIS2006-03438, by the Generalitat Valenciana contract ACOMP07/302, by Junta de Andalucía contract FQM0225 and by the EU Integrated Infrastructure Initiative Hadron Physics Project contract RII3-CT-2004-506078.

[1] Nuint'07 Proc., AIP Conf. Proc. 967 (2007), eds. G. P. Zeller, J. G. Morfin, F. Cavanna.

[2] A. A. Aguilar-Arevalo et al. [MiniBooNE Coll.], Phys. Rev. Lett. 100 (2008) 032301.

[3] J. M. Link, AIP Conf. Proc. 967 (2007) 151.

[4] J. Nieves, J. E. Amaro and M. Valverde, Phys. Rev. C 70 (2004) 055503 [Erratum-ibid. C 72 (2005) 019902].

[5] J. E. Amaro et al., Phys. Rev. C 71 (2005) 015501.

[6] T. Leitner, L. Alvarez-Ruso and U. Mosel, Phys. Rev. C 73 (2006) 065502.

[7] O. Benhar, N. Farina, H. Nakamura, M. Sakuda and R. Seki, Phys. Rev. D 72 (2005) 053005.

[8] J. Nieves, M. Valverde and M. J. Vicente Vacas, Phys. Rev. C 73 (2006) 025504.

[9] R. C. Carrasco and E. Oset, Nucl. Phys. A 536 (1992) 445.

[10] R. C. Carrasco, M. J. Vicente Vacas and E. Oset, Nucl. Phys. A 570 (1994) 701.

[11] A. Gil, J. Nieves and E. Oset, Nucl. Phys. A 627 (1997) 543.

[12] L. Alvarez-Ruso, L. S. Geng, S. Hirenzaki and M. J. Vicente Vacas, Phys. Rev. C 75 (2007) 055501.

[13] L. Alvarez-Ruso, L. S. Geng and M. J. Vicente Vacas, Phys. Rev. C 76 (2007) 068501.

[14] L. Alvarez-Ruso et al., AIP Conf. Proc. 967, 201 (2007).

[15] N. G. Kelkar, E. Oset and P. Fernandez de Cordoba, Phys. Rev. C 55 (1997) 1964.

[16] S. K. Singh, M. Sajjad Athar and S. Ahmad, Phys. Rev. Lett. 96, 241801 (2006).

[17] E. Hernandez, J. Nieves and M. Valverde, Phys. Rev. D 76 (2007) 033005.

[18] T. Kitagaki et al., Phys. Rev. D 42 (1990) 1331.

[19] G. M. Radecky et al., Phys. Rev. D 25 (1982) 1161 [Erratum-ibid. D 26 (1982) 3297].

[20] E. A. Paschos, A. Kartavtsev and G. J. Gounaris, Phys. Rev. D 74 (2006) 054007.

[21] C. Garcia-Recio et al., Nucl. Phys. A 526 (1991) 685.

[22] J. Nieves, E. Oset and C. Garcia-Recio, Nucl. Phys. A 554 (1993) 554.

[23] L. Alvarez-Ruso, S. K. Singh and M. J. Vicente Vacas, Phys. Rev. C 59 (1999) 3386.

[24] J. Liu, N. C. Mukhopadhyay and L. s. Zhang, Phys. Rev. C 52 (1995) 1630.

[25] T. Sato, D. Uno and T. S. H. Lee, Phys. Rev. C 67 (2003) 065201.

[26] O. Lalakulich, E. A. Paschos and G. Piranishvili, Phys. Rev. D 74 (2006) 014009.

[27] D. Barquilla-Cano, A. J. Buchmann and E. Hernandez, Phys. Rev. C 75 (2007) 065203.

[28] L. S. Geng et al., arXiv:0801.4495 [hep-ph].

[29] N. C. Mukhopadhyay, M. J. Ramsey-Musolf, S. J. Pollock, J. Liu and H. W. Hammer, Nucl. Phys. A 633 (1998) 481. 